\title{
Synthesis, transport and magnetic properties of Ba-Co-Ge clathrates
}

\author{
Ali A. Sirusi · Joseph H. Ross, Jr. \\ Cite as: J. Electronic Mater. 45, 1094-1100 (2016). Published version (C) 2015 The Minerals, Metals \& Materials \\ Society.
}

link to published version: http://dx.doi.org/10.1007/s11664-015-4259-6

Received: date / Accepted: date

\begin{abstract}
Ba-Co-Ge intermetallic clathrates were synthesized in both type-I and chiral-type crystal structures, to investigate thermoelectric and physical properties. Seebeck coefficients, thermal conductivities, electrical resistivities, and Hall coefficients were measured, as well as specific heat and magnetic susceptibility. TypeI $\mathrm{Ba}_{8} \mathrm{Co}_{x} \mathrm{Ge}_{46-y}$ was formed with a large number of spontaneous vacancies, similar to a Zintl condition, but without the vacancy ordered superstructure of $\mathrm{Ba}_{8} \mathrm{Ge}_{43}$. However the vacancies for this composition do not moderate the carrier density as expected from Zintl electron balancing. Instead the physical properties point to a complex Fermi surface property with a large effective carrier density, a behavior consistent with other materials close to $\mathrm{Ba}_{8} \mathrm{Ge}_{43}$. In the case of chiral samples, Co substitution strongly suppresses only the lower of the two structural transformations. Susceptibility and specific heat measurements, coupled with the measured transport properties, demonstrate that the electron densities of states near the Fermi energy change very little in the transformations, a significant reduction in the effect of these transitions compared to the case of unsubstituted $\mathrm{Ba}_{6} \mathrm{Ge}_{25}$.
\end{abstract}

Keywords Clathrates - Thermoelectric materials · Magnetization · Transport properties

\footnotetext{
A. Sirusi

Department of Physics and Astronomy, Texas A\&M University, College Station, TX 77843, USA.

Tel.: +1-979-845-7823

Fax: +1-979-845-2590

E-mail: ali.sirusi@gmail.com

J. Ross

Department of Physics and Astronomy and Department of Materials Science and Engineering, Texas A\&M University, College Station, TX 77843, USA.
}

\section{Introduction}

Group IV intermetallic clathrates have gained great attention due to their outstanding thermoelectric performance, superconductivity, magnetic ordering, glass-like thermal conductivity, and unusual Kondo effects for over a decade [1-5]. The intermetallic clathrates can have very low thermal conductivities of the same order as amorphous materials. Phonon scattering by rattling centers (guest atoms) [1,6] and/or the change of the characteristic propagative phonon modes $[7,8]$ contribute to the low thermal conductivity. Due to these properties it is suggested that properties of some clathrates may obey the phonon-glass electron-crystal concept [9].

The intermetallic clathrate compounds can also follow the Zintl rule [10] in which compositions having a balanced-electron count can be more chemically stable, and tend to be semiconductors. In Zintl-balanced type-I clathrates (for example, $\mathrm{Ba}_{8} \mathrm{Ga}_{16} \mathrm{Ge}_{30}$ ) the $\mathrm{Ba}$ atoms donate 16 electrons to the framework and with three electrons each, 16 atoms of Ga fulfill the balancedelectron count of 4 per atom. However, most clathrates show metallic transport due to heavy doping in partially off stoichiometric compositions.

The type-I clathrate structure has one $M_{8} X_{46}$ formula unit per cubic cell, with the framework consisting of two small cages (dodecahedra) and six large ones (tetrakaidecahedra) [6]. The alternative chiral clathrate structure (sometimes designated type-IX), has four $M_{6} X_{25}$ formula units per cell. In these cases $M$ is a guest atom such as $\mathrm{Ba}$, and the $X$ atoms making up the framework are largely $\mathrm{Ge}, \mathrm{Si}$, or $\mathrm{Sn}$. The chiral structure can be related to the type-I by replacing two pairs of Ge by Ba atoms [11] as a result of which three distinct Ba sites exist. $\mathrm{Ba}_{6} \mathrm{Ge}_{25}$ has a high carrier density (around $10^{22}$ $\mathrm{cm}^{-3}$ ), not suitable for efficient thermoelectrics [12]. 
However note that Zintl balance can also be obtained in the chiral structure, for example by In substitution of four per formula unit [13], yielding the composition $\mathrm{Ba}_{6} \mathrm{In}_{4} \mathrm{Ge}_{21}$.

A parameter used to compare the thermoelectric efficiency is the dimensionless figure of merit, $z T$, defined at temperature $T$ as $z T=\frac{S^{2} \sigma T}{\kappa}$, where $S$ is the Seebeck coefficient, $\sigma$ is the electrical conductivity, and $\kappa$ is the thermal conductivity. Because these three quantities can be adjusted by changing the carrier density, atoms such as $\mathrm{Al}, \mathrm{Ga}$, In, or transition metals can be substituted in the framework to boost $z T$, even in chiral clathrates that are normally strongly metallic $[14,15]$. In addition, $\mathrm{Ba}_{6} \mathrm{Ge}_{25}$ exhibits very interesting features at low temperature such as first-order phase transitions, superconductivity, and ferromagnetism [12,16,17].

Co-doped clathrates have not been investigated as heavily as $\mathrm{Ba}_{8} \mathrm{Ga}_{16} \mathrm{Ge}_{30}$ due in part to difficulties in the syntheses. In this paper we report the physical properties of type-I and chiral Ba-Co-Ge compounds from 2 $\mathrm{K}$ to $300 \mathrm{~K}$.

\section{Sample Preparation and Experimental Methods}

Several samples of Ba-Co-Ge clathrates were prepared from pure elements [Ge (pieces, 5N), Ga (ingot, $4 \mathrm{~N}$ ), and $\mathrm{Ba}(\mathrm{rod}, 2 \mathrm{~N} 5)]$ with excess (4\%) barium to compensate for evaporation, and arc melted in an argon environment following by annealing in a BN crucible in an evacuated sealed quartz tube for one week. Three of these polycrystalline ingot were selected for further study as described below. The type-I sample was annealed at $800{ }^{\circ} \mathrm{C}$ followed by quenching in water and the chiral samples were annealed at $750{ }^{\circ} \mathrm{C}$ and slowly cooled to room temperature. Powder x-ray diffraction (XRD) measurements were performed at room temperature using a Bruker D8 X-ray Powder Diffractometer, and Rietveld refinement was performed using EXPGUI $[18,19]$. Wavelength dispersive spectroscopy (WDS) measurements were performed on at least four points on the samples in a Cameca SX50 equipped with 4 wavelengthdispersive X-ray spectrometers. Resistivity, Hall coefficient, Seebeck coefficient, heat capacity, and thermal conductivity measurements were performed in a Quantum Design Physical Property Measurement System. A Quantum Design SQUID magnetometer was used to measure magnetic properties.

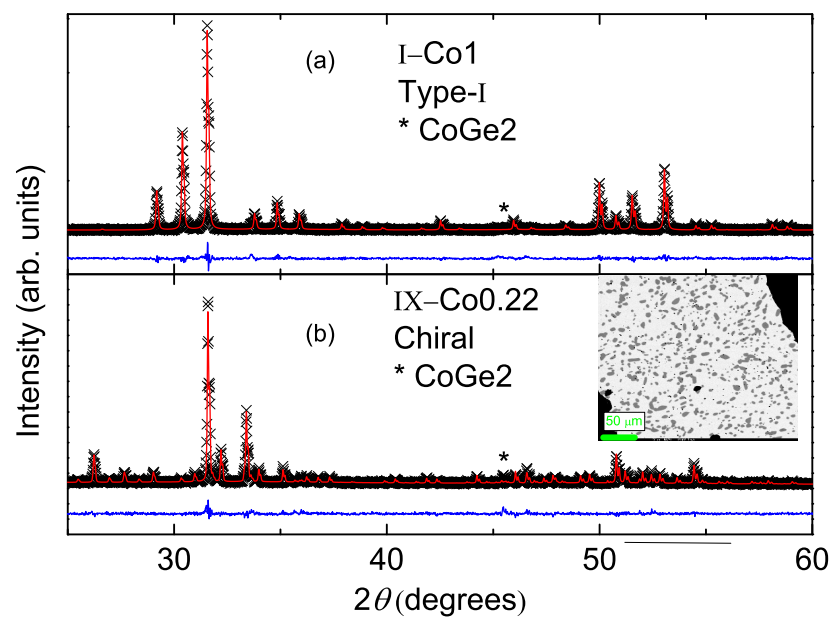

Fig. 1 X-ray data with Rietveld refinement profile (red lines) and the difference (blue lines) for a) $\mathrm{Ba}_{8} \mathrm{Co}_{3} \mathrm{Ge}_{43}$ (sample I-Co1) b) $\mathrm{Ba}_{6} \mathrm{Co}_{5} \mathrm{Ge}_{20}$ (sample IX-Co0.22), showing also a back-scattered electron image.

Table 1 Sample compositions and lattice constants extracted from WDS and powder XRD, respectively, with carrier densities $(n)$ from Hall measurements (with minus sign denoting n-type).

\begin{tabular}{lccccl} 
nominal & $\mathrm{Ba}$ & $\mathrm{Co}$ & $\mathrm{Ge}$ & $\begin{array}{c}a \\
(\mathrm{~nm})\end{array}$ & $\begin{array}{l}n \\
\left(\mathrm{~cm}^{-3}\right)\end{array}$ \\
\hline $\mathrm{Ba}_{8} \mathrm{Co}_{3} \mathrm{Ge}_{43}$ (I-Co1) & 8.00 & 0.96 & 42.41 & 1.0671 & $-4 \times 10^{21}$ \\
$\mathrm{Ba}_{6} \mathrm{Co}_{5} \mathrm{Ge}_{20}$ (IX-Co0.22) & 5.94 & 0.22 & 24.77 & 1.4144 & $-8 \times 10^{21}$ \\
$\mathrm{Ba}_{6} \mathrm{Co}_{2} \mathrm{Ge}_{23}$ (IX-Co0.27) & 6.00 & 0.27 & 24.71 & 1.4542 & $4 \times 10^{21}$
\end{tabular}

\section{Experimental results}

Powder XRD measurements on the nominal $\mathrm{Ba}_{8} \mathrm{Co}_{3} \mathrm{Ge}_{43}$ sample showed the material to have the type-I clathrate structure (space group $\operatorname{Pr} \overline{3} n$, with Ba in cage-centered positions). Nominal $\mathrm{Ba}_{6} \mathrm{Co}_{2} \mathrm{Ge}_{23}$ and $\mathrm{Ba}_{6} \mathrm{Co}_{5} \mathrm{Ge}_{20}$ samples were shown to have the chiral structure (space group $P 4_{1} 32$ ) with small extra phases, $\mathrm{CoGe}_{2}$ and $\mathrm{Ge}$. Fig. 1 shows the XRD results and fits for the $\mathrm{Ba}_{8} \mathrm{Co}_{3} \mathrm{Ge}_{43}$ and $\mathrm{Ba}_{6} \mathrm{Co}_{5} \mathrm{Ge}_{20}$ samples. See Table 1 for lattice constants from the XRD refinements.

The detailed compositions of all samples from WDS are given in Table 1. The composition of the type-I sample is $\mathrm{Ba}_{8} \mathrm{Co}_{1} \mathrm{Ge}_{42.5}$. This composition has a vacancy concentration of 2.6 per cell, slightly reduced from that of unsubstituted $\mathrm{Ba}_{8} \mathrm{Ge}_{43} \square_{3}$, which has a superstructure with space group Ia $\overline{3} \mathrm{~d}$, however we did not observe superstructure peaks for the substituted material. The $\mathrm{Ba}_{6} \mathrm{Co}_{0.27} \mathrm{Ge}_{24.71}$ and $\mathrm{Ba}_{5.94} \mathrm{Co}_{0.22} \mathrm{Ge}_{24.77}$ chiral clathrates have no framework vacancies according to the WDS measurements. We denoted the type-I sample as I-Co1, and the chiral (type-IX) samples as IX-Co0.27 and IX-Co0.22. The lattice constants of IXCo0.22 and IX-Co0.27, also in Table 1, are slightly reduced from the reported value [12] $a=1.45564(2) \mathrm{nm}$ 
for $\mathrm{Ba}_{6} \mathrm{Ge}_{25}$. On the other hand the I-Co1 lattice constant is slightly larger (when doubled) than of the [20] type-I $\mathrm{Ba}_{8} \mathrm{Ge}_{43} \square_{3}$ which has a $2 \times 2 \times 2$ supercell with $a=2.1307(1) \mathrm{nm}$. These results are consistent with those of Ref. [21], and also in line with results for typeI Ba-Ge clathrate substitution by $\mathrm{Cu}$ and $\mathrm{Ni}$ [22-24], which also induce an expansion of the lattice.

Note that the amount of Co substituted in the typeI sample is smaller than the maximum reported in reference [21], where it was found that the solubility of Co in the type-I and chiral structures can be up to 2.5 and 1 per unit cell, respectively. The samples measured here came from a number of trial syntheses, and these compositions were the largest Co concentrations yielding continuous clathrate phases allowing transport measurements to be performed. While in $\mathrm{Cu}$ type-I clathrates the Zintl condition is found to strongly stabilize the composition $[22,23,25]$, Ni clathrates allow more variation in composition [24,25], an apparent indication that the stabilization is lessened. For Co substitution (with Zintl condition corresponding to 2.5 per cell for type I) this trend of apparently lower stability for the Zintl composition continues and competes with $\mathrm{CoGe}_{2}$ phase formation [21]. Here, a portion of the Co remained in the ingots as $\mathrm{CoGe}_{2}$; for example for the Co0.22 sample which had the largest initial Co concentration, a back-scattered electron image is shown in Fig. 1. In other samples the second phase is correspondingly reduced (see star positions in the XRD spectra, Fig. 1). With further increased substitution, approaching the Zintl compositions, the clathrate phases were found to be nonuniform and unsuitable for measurements of physical properties.

The Hall coefficient $R_{H}$ was obtained by measuring the Hall resistivity $\left(\rho_{H}\right)$ versus field $\left(\rho_{H}=R_{H} B\right)$. Assuming a one-band model, the carrier density, $n$, was determined according to $n=1 /\left(R_{H} e\right)$. Results are given in Table 1 . The type-I carrier density is affected by vacancies, formed spontaneously in many clathrates $[2,26]$ for electron balance. This follows the Zintl condition, promoting a 4-bonded framework as noted earlier. In the observed I-Co1 composition, eight $\mathrm{Ba}^{2+}$ ions can be considered to donate a total of 16 electrons per formula unit, each Co requires 5 electrons to satisfy 4 framework bonds (with d-shells filled, consistent with the diamagnetic behavior described below), and 4 electrons per vacancy are needed to fill dangling bonds. The observed I-Co1 composition (Table 1) includes 2.6 vacancies per cell, so 15.3 electrons per formula unit are needed to complete the $\mathrm{sp}^{3}$ framework, leaving 0.7 electrons to go into the conduction band. However, the Hall results correspond to a larger apparent concentration of about 4.8 electrons per cell. This is similar to results for
$\mathrm{Ba}_{8} \mathrm{Ni}_{x} \mathrm{Ge}_{46-y}$ [24], in which for small $x$ multiple vacancies per cell were obtained, yet with a very large carrier density comparable to what is observed here. It appears that the Fermi-level electronic structure in the present case, as well as for low-Ni substitution, is characterized by multiple band crossings, as evidenced originally in [27] $\mathrm{Ba}_{8} \mathrm{Ge}_{43}$, and although multiple vacancies are formed this does not serve to reduce significantly the carrier density as might be expected.

With no vacancies in the chiral samples according to the measured compositions, a similar calculation based on framework bonding [13] yields about 12 electrons per cell above the Zintl condition for both cases, a strongly metallic situation as expected for such materials. The large observed Hall carrier densities are therefore not surprising, however similar to the above result, a multiband condition at the Fermi energy is also expected for these materials, so that the Hall carrier densities do not necessarily reflect the number of carriers above the Zintl condtion. Indeed, calculations have shown [28] an electronic density of states for $\mathrm{Ba}_{6} \mathrm{Ge}_{25}$ characterized by a number of sharp peaks in the vicinity of the Fermi energy, a condition which may explain the observed $n$ and $p$ type behavior associated with small changes in composition.

Figure 2 shows resistivities from $2 \mathrm{~K}$ to room temperature. For type-I the magnitude and behavior is similar to that of $\mathrm{Ba}_{8} \mathrm{Ge}_{43}$ [27] and low- $x \mathrm{Ba}_{8} \mathrm{Ni}_{x} \mathrm{Ge}_{46-y}$ [24], reinforcing the similar electronic behavior of these materials as noted above. The resistivities of the chiral samples are generally metallic-like, however they increase with deceasing temperature below $120 \mathrm{~K}$, due to the low temperature phase transition. There is also a small slope change near $220 \mathrm{~K}$ corresponding to the second phase transition (compared to $215 \mathrm{~K}$ in unsubstituted $\mathrm{Ba}_{6} \mathrm{Ge}_{25}$ ), seen more distinctly in other properties as described below. Sample IX-Co0.22 with lower Co concentration shows the trend more clearly. Since the resistivities show thermal hysteresis below $120 \mathrm{~K}$, these must correspond to first-order phase transitions. This low-temperature anomaly is suppressed to lower temperatures compared to the transition observed [12] near $180 \mathrm{~K}$ in $\mathrm{Ba}_{6} \mathrm{Ge}_{25}$, while the other transition occurs at about the same temperature but with a much smaller effect on transport properties. Qualitatively, this is similar to the effect of Eu substitution [12] for $\mathrm{Ba}$ in $\mathrm{Ba}_{6} \mathrm{Ge}_{25}$, although Co substituents are nonmagnetic, and substitute on the framework rather than in guest sites.

The lattice thermal conductivities $\left(\kappa_{L}\right)$ and Seebeck coefficients $(S)$ of the I-Co1 and IX-Co0.27 samples are shown in Fig. 3. For $\kappa_{L}$ we subtracted the electronic contribution from the measured values using the Wiedemann-Franz law. $\kappa_{L}$ of I-Co1 shows a crystal-like 


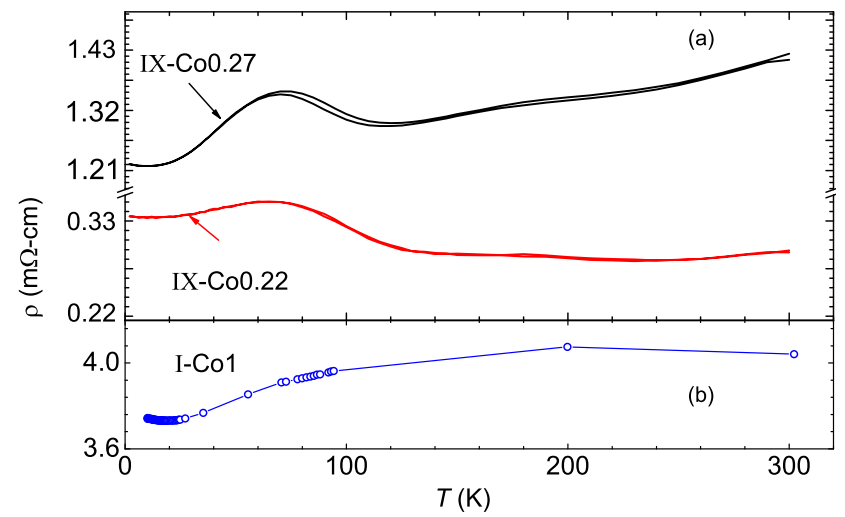

Fig. 2 Resistivities of Ba-Co-Ge clathrates as a function of temperature a) IX-Co0.27 and IX-Co0.22 measured both for heating and cooling b) I-Co1.

peak and reaches $1.7 \mathrm{~W} /(\mathrm{K}-\mathrm{m})$ at room temperature. Thus with Co substitution the phonon mobility for this sample remains high, although reduced from that of $\mathrm{Ba}_{8} \mathrm{Ge}_{43}$ [20]. While vacancy scattering will affect the thermal conductivity, it was recently indicated that the order or disorder of the vacancies may make surprisingly little difference [29]. However note that the thermal conductivity is larger than that of $\mathrm{Ba}_{8} \mathrm{Ga}_{16} \mathrm{Ge}_{30}$ presumably due to reduced alloy scattering [6].

The IX-Co0.27 sample does not exhibit a crystallinetype peak, and the magnitude of $\kappa_{L}$, reaching 1.48 $\mathrm{W} / \mathrm{K}-\mathrm{m}$ at room temperature, qualifies as glass-like behavior. Slope changes are also seen in its $\kappa_{L}$ at the temperatures of both phase transitions. The Seebeck coefficient for IX-Co0.27 is negative in spite of it being p-type at room temperature, presumably due to multiband behavior as noted above. Its magnitude, $S=-20$ $\mu \mathrm{V} / \mathrm{K}$ at room temperature, is relatively small consistent with the metallic behavior.

A notable feature of the Seebeck coefficient of IXCo0.27 is the changes in slope at about $90 \mathrm{~K}$ and $230 \mathrm{~K}$, consistent with the transitions seen in resistivity. This is shown by the straight lines in Fig. 3(b). The fitted slopes change from $-0.076 \mu \mathrm{V} / \mathrm{K}^{2}$ at low temperatures to $-0.056 \mu \mathrm{V} / \mathrm{K}^{2}$ between 120 and $220 \mathrm{~K}$, and $-0.113 \mu \mathrm{V} / \mathrm{K}^{2}$ above $240 \mathrm{~K}$.

The heat capacity of IX-Co0.27 is shown in Fig. 4. The electronic contribution, $\gamma$, was determined by fitting the low temperature data to a straight line according to $C_{p} / T=\gamma+\beta T^{2}$. Results are $\gamma=23 \mathrm{~mJ} / \mathrm{molK}^{2}$ for IX-Co0.27, and $29 \mathrm{~mJ} / \mathrm{molK}^{2}$ for IX-Co0.22, where mole values refer to the $\mathrm{Ba}_{6} \mathrm{X}_{25}$ formula unit, while a similar measurement for I-Co1 yields $\gamma=42 \mathrm{~mJ} / \mathrm{molK}^{2}$ (per mole $\mathrm{Ba}_{8} \mathrm{X}_{46}$ )

To model the results, we fitted the chiral data using Debye and Einstein models. Since the low-temperature phase transition is suppressed to near $100 \mathrm{~K}$ by Co sub-

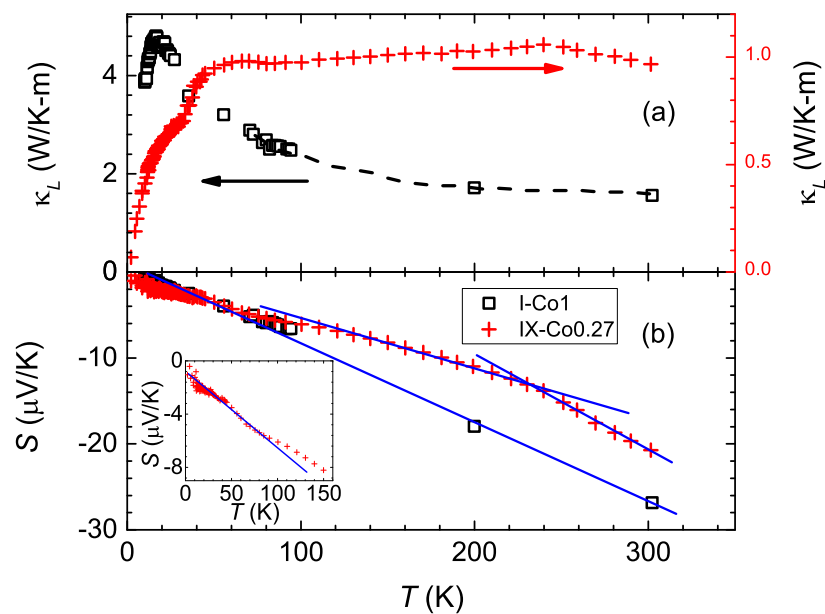

Fig. 3 (a) Thermal conductivity and (b) Seebeck coefficient vs temperature for I-Co1 (open squares) and IX-Co0.27 (open circles). Inset shows Seebeck coefficient below $160 \mathrm{~K}$ for IXCo0.27 sample. Solid lines are fitted straight lines.

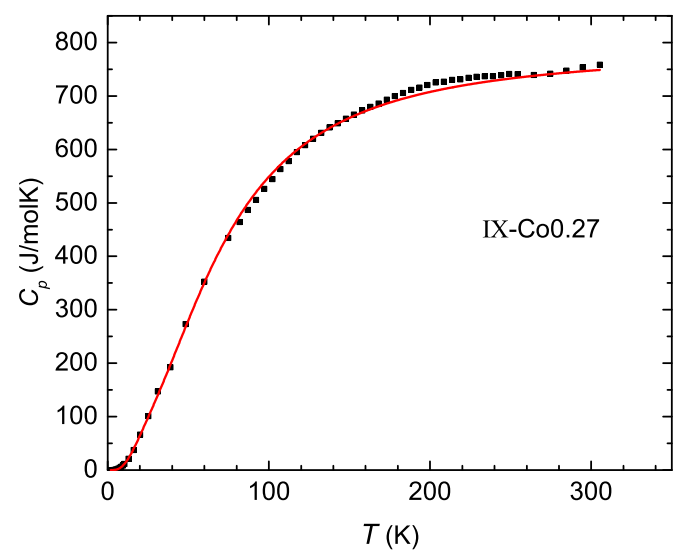

Fig. 4 Heat capacity $\left(C_{P}\right)$ for sample IX-Co0.27. Solid curve fit to the model described in text. Data are normalized per mole formula unit of $\mathrm{Ba}_{6} \mathrm{X}_{25}$.

stitution, this fit runs through the transformations, thus provides only a qualitative measure of the localized oscillator behavior, however the fitted curve allows the effects of transformations in specific heat to be seen more clearly. We considered two Einstein terms $\left(C_{E}\right)$ with parameters $\Theta_{E 1}$ and $\Theta_{E 2}$, one Debye term $\left(C_{D}\right)$ with parameter $\Theta_{D}$, plus the electronic term $C_{e l}=\gamma T$, with $\gamma$ from the low-temperature fit. These are:

$$
\begin{gathered}
C_{E}=3 N_{E} R\left(\frac{\Theta_{E}}{T}\right)^{2} \frac{e^{\Theta_{E} / T}}{\left(e^{\Theta_{E} / T}-1\right)^{2}}, \\
C_{D}=9 N_{D} R\left(\frac{T}{\Theta_{D}}\right)^{3} \int_{0}^{\Theta_{D} / T} \frac{x^{4} e^{x} d x}{\left(e^{x}-1\right)^{2}},
\end{gathered}
$$

where $N_{D}$ and $N_{E}$ are the number of oscillators, which should correspond to the number of framework atoms and guest atoms, respectively. 


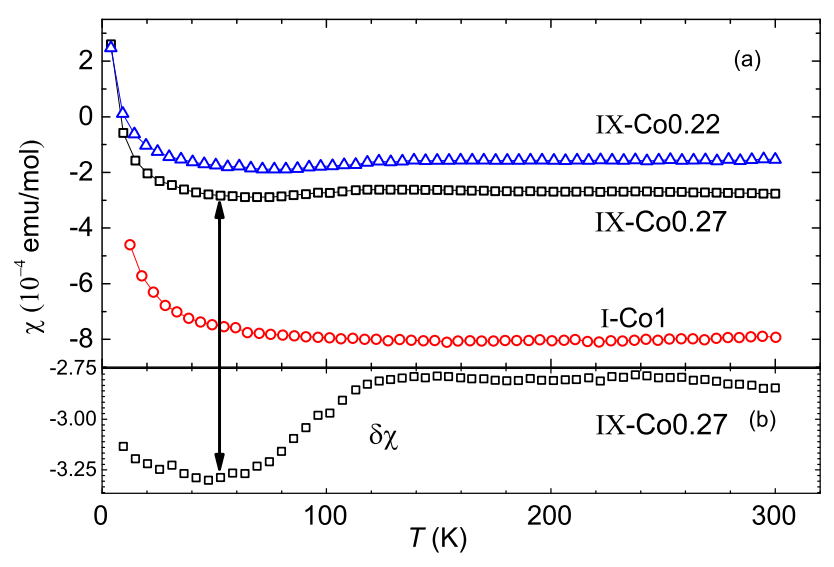

Fig. 5 Temperature dependence of magnetic susceptibility of (a) IX-Co0.27 (squares), IX-Co0.22 (triangles) and ICo1(open circles). (b) IX-Co0.27 results after removing small Curie paramagnetism as explained in the text. Values per mole formula unit $\left(\mathrm{Ba}_{6} \mathrm{X}_{25}\right.$, or $\left.\mathrm{Ba}_{8} \mathrm{X}_{46}\right)$.

Table 2 Heat capacity fitting parameters.

\begin{tabular}{ccccc} 
sample & $\begin{array}{c}\Theta_{D} \\
(\mathrm{~K})\end{array}$ & $\begin{array}{c}\Theta_{E 1} \\
(\mathrm{~K})\end{array}$ & $\begin{array}{c}\Theta_{E 2} \\
(\mathrm{~K})\end{array}$ & $\begin{array}{c}\gamma \\
\left(\mathrm{mJ} / \mathrm{mol} \mathrm{K}^{2}\right)\end{array}$ \\
\hline IX-Co0.27 & 308 & 84 & 43 & 23 \\
IX-Co0.22 & 301 & 80 & 60 & 29
\end{tabular}

Table 2 shows the parameters from least squares fitting. For the chiral structures we used $N_{D}=25$ $N_{E 1}=5, N_{E 2}=1$. Although we have three Ba sites, this corresponds to combining two guest sites to make $N_{E 1}$. The fitted curve [Fig. 4] deviates slightly as expected in the region of the low-temperature transition near $100 \mathrm{~K}$, while the high-temperature transition is more obvious in the results.

Figure 5(a) shows the magnetic susceptibilities measured at 5000 Oe. All samples exhibit diamagnetism, a normal case for most intermetallic clathrates. I-Co1 has $\chi=-7.9 \times 10^{-4}(\mathrm{emu} / \mathrm{mol})$ at room temperature, compared to the calculated core diamagnetism, $-5.2 \times$ $10^{-4}(\mathrm{emu} / \mathrm{mol})$. In all cases the small Curie-like lowtemperature upturn corresponds to very dilute moments, rather than intrinsic magnetism. This was confirmed by $M-H$ measurements at low temperatures; for example in sample IX-Co0.27 the $M-H$ results (not shown) could be fitted almost entirely due to isolated moments with effective paramagnetic moment $6.1 \mu_{B}$ and concentration $5 \times 10^{-4}$ per formula unit. Subtracting this contribution, we obtain the net result $(\delta \chi)$ shown in Fig. 5(b), where the effects of the two transitions can be seen more clearly as small slope changes in $\chi$. Therefore, the large diamagnetism is suggested to be due to structural diamagnetism of the clathrates [12].

\section{Analysis and discussion}

Assuming that the electron-phonon enhancement is small, the electronic heat capacity term is [30], $\gamma=\left(k_{B} \pi\right)^{2} g\left(E_{F}\right) / 3$. Based on this relation and the results quoted above we obtain the low-temperature densities of states $g\left(E_{F}\right)=$ 12.3 and 9.9 states/(eV-mole $\left.\mathrm{Ba}_{6} \mathrm{X}_{25}\right)$ for IX-Co0.22 and IX-Co0.27, respectively. These are similar to results for the low-temperature $\mathrm{Ba}_{6} \mathrm{Ge}_{25}$ phase from DFT calculations [31], though a factor of about 4 larger than an experimental estimate for $\mathrm{Ba}_{6} \mathrm{Ge}_{25}$ obtained by other means [32].

From $g\left(E_{F}\right)$ we also obtain the low temperature Pauli contribution to the susceptibility using $\chi_{\text {Pauli }}=$ $\mu_{B}^{2} g\left(E_{F}\right)$, giving $\chi_{\text {Pauli }}=4.0 \times 10^{-4}$ and $3.2 \times 10^{-4}$ $\mathrm{emu} /\left(\right.$ mole $\left.\mathrm{Ba}_{6} \mathrm{X}_{25}\right)$ for IX-Co0.22 and IX-Co0.27 respectively. The difference in calculated values is comparable to the observed difference, $\Delta \chi \approx 1.2 \times 10^{-4}$ $\mathrm{emu} /\left(\right.$ mole $\left.\mathrm{Ba}_{6} \mathrm{X}_{25}\right)$, nearly independent of temperature.

We thus assume that the diamagnetic background term $\left(\chi_{\text {dia }}\right)$ is the same for both chiral compositions, with the observed temperature dependences due to changes in $\chi_{\text {Pauli }}$. (We assume the Landau term also to be temperature independent; a similar assumption was made in Ref. [32].) Starting with the low temperature baseline susceptibility for IX-Co0.27 (Fig. 5(b)) and extrapolating to $\chi_{\text {Pauli }}=0$ we obtain $\chi_{\text {dia }}=-6.4 \times$ $10^{-4} \mathrm{emu} /\left(\right.$ mole $\left.\mathrm{Ba}_{6} \mathrm{X}_{25}\right)$, comparable to the value $-8 \times$ $10^{-4} \mathrm{emu} / \mathrm{mole}$ extracted in Ref. [32] for unsubstituted $\mathrm{Ba}_{6} \mathrm{X}_{25}$.

The small observed changes in $\chi$ at the phase transformations in the chiral samples indicate that $g\left(E_{F}\right)$ does not change substantially at the phase transitions. This is in contrast to results [33,32] for unsubstituted $\mathrm{Ba}_{6} \mathrm{Ge}_{25}$. The changes in $\chi$ can be seen most clearly in the difference curve for IX-Co0.27, Fig. 5(b): the low-temperature transition is accompanied by a change $\Delta \chi \approx-5 \times 10^{-5}\left(\mathrm{emu} / \mathrm{mole} \mathrm{Ba}_{6} \mathrm{X}_{25}\right)$, or about $10 \%$ of the low-temperature $\chi_{\text {Pauli }}$ value. This corresponds to a $10 \%$ change in $g\left(E_{F}\right)$, in contrast with the factor of 4 reduction estimated [33,32] for $\mathrm{Ba}_{6} \mathrm{Ge}_{25}$. This small change is in line with the much smaller effect observed in resistivity. Thus this transformation is reduced in temperature in the Co-substituted material, and is also accompanied by a greatly reduced effect on the electronic density of states.

The loss of carriers in the structural distortion in $\mathrm{Ba}_{6} \mathrm{Ge}_{25}$ has been connected through crystallography to breaking of bonds [16], and thus the results seen here are presumably due to a reduced-magnitude displacement of $\mathrm{Ba}$ ions associated with the substituted framework. Similar effects may accompany Eu substi- 
tution [12], which as we noted produces quite similar changes in the observed transport behavior. Indeed, the observed resistivity behavior and also the reduction in as well as broadening of the low temperature phase transformation observed here is quite similar to the results displayed [12] for a $\mathrm{Eu}_{0.8}$ substituted sample, results which were explained as consistent with a chemical pressure effect due to the decreased lattice constant, as also observed for Co substitution.

Per framework atom, the measured $\gamma$ for I-Co1 is quite close to that of the chiral-materials, which reinforces its strongly metallic behavior of this composition as also evidenced by the transport results. As noted above, comparing the similar transport results for $\mathrm{Ba}_{8} \mathrm{Ge}_{43}$ indicates that the Co-substitued composition presumably also exhibits complicated bands cutting $E_{F}$ leading to multiple carrier types. The observed $\gamma$ can also be used to estimate the Pauli susceptibility of the type-I composition. Using the relationships described above yields $\chi_{\text {Pauli }}=5.8 \times 10^{-4}\left(\mathrm{emu} / \mathrm{mol} \mathrm{Ba} 8 \mathrm{X}_{46}\right)$ for I-Co1. Subtracting this from the measured result we obtain a bare diamagnetic contribution of $\chi_{\text {dia }}=$ $-14 \times 10^{-4} \mathrm{emu} /\left(\right.$ mole $\left.\mathrm{Ba}_{8} \mathrm{X}_{46}\right)$, more diamagnetic by about $-9 \times 10^{-4} \mathrm{emu} /\left(\right.$ mole $\left.\mathrm{Ba}_{8} \mathrm{X}_{46}\right)$ than the calculated core diamagnetism. The origin for this large contribution remains unclear, although sometimes this contribution is attributed to ring currents in the clathrate framework. Note also that per framework atom, the results for the two structures are similar: we find that with $\chi_{\text {Pauli }}$ removed, $\chi_{\text {dia }}=-2.6 \times 10^{-5} \mathrm{emu} /($ mole-Xatom) for both chiral compositions and $\chi_{\text {dia }}=-3.1 \times$ $10^{-5} \mathrm{emu} /($ mole-X-atom) for the type-I. Results quoted [32] above for $\mathrm{Ba}_{6} \mathrm{Ge}_{25}$ correspond to the similar value, $\chi_{\text {dia }}=-3.1 \times 10^{-5} \mathrm{emu} /($ mole-X-atom $)$. Thus it is may be that a ring current or related electronic feature per framework atom underlies this behavior.

\section{Conclusions}

Investigating Co-substituted Ba-Ge clathrates, we synthesized several compositions and measured resistivity, Hall coefficient, magnetic susceptibility, specific heat, thermal conductivity, and Seebeck coefficient. Type-I $\mathrm{Ba}_{8} \mathrm{Co}_{x} \mathrm{Ge}_{46-y}$ exhibits vacancy formation similar to unsubstituted $\mathrm{Ba}_{8} \mathrm{Ge}_{43}$, but with the vacancy-ordering suppressed. Similar to other compositions close to $\mathrm{Ba}_{8} \mathrm{Ge}_{4}$ however, the vacancies appear not to moderate the carrier density, and instead the physical properties point to a complex Fermi surface property with a large effective carrier density. In chiral $\mathrm{Ba}_{6} \mathrm{Co}_{x} \mathrm{Ge}_{25-x}$ clathrates, the low-temperature structural transition transformation has a transition temperature that is significantly reduced, while the upper transformation temperature remains nearly unchanged. However the magnetic, calorimetric, and transport behavior demonstrate that the effect of these transitions on the electron density of states is strongly suppressed, in contrast to the behavior of unsubstituted material.

Acknowledgements This work was supported by the Robert A. Welch Foundation, Grant no. A-1526.

\section{References}

1. G.S. Nolas, J.L. Cohn, G.A. Slack, S.B. Schujman, Appl. Phys. Lett. 73, 178 (1998)

2. M. Christensen, S. Johnsen, B.B. Iversen, Dalton Trans. 39, 978 (2010)

3. B.C. Sales, B.C. Chakoumakos, R. Jin, J.R. Thompson, D. Mandrus, Phys. Rev. B 63, 245113 (2001)

4. J.H. Ross, Jr., Y. Li, in Nanoscale Magnetic Materials and Applications, ed. by J.P. Liu, E. Fullerton, O. Gutfleisch, D. Sellmyer (Springer, New York, 2009), chap. 4, p. 105

5. A. Prokofiev, A. Sidorenko, K. Hradil, M. Ikeda, R. Svagera, M. Waas, H. Winkler, K. Neumaier, S. Paschen, Nat. Mater. 12, 1096 (2013)

6. T. Takabatake, K. Suekuni, T. Nakayama, E. Kaneshita, Rev. Mod. Phys. 86, 669 (2014)

7. S. Pailhès, H. Euchner, M. Giordano, V., R. Debord, A. Assy, S. Gomès, A. Bosak, D. Machon, S. Paschen, M. de Boissieu, Phys. Rev. Lett. 113, 025506 (2014)

8. T. Tadano, Y. Gohda, S. Tsuneyuki, Phys. Rev. Lett. 114, $095501(2015)$

9. G. Slack, in CRC Handbook of Thermoelectrics, ed. by D.M. Rowe (CRC Press, 1995)

10. E. Zintl, Angew. Chem. 52, 1 (1939)

11. H. Fukuoka, K. Iwai, S. Yamanaka, H. Abe, K. Yoza, L. Häming, J. Solid State Chem. 151, 117 (2000)

12. S. Paschen, V.H. Tran, M. Baenitz, W. Carrillo-Cabrera, Y. Grin, F. Steglich, Phys. Rev. B 65, 134435 (2002)

13. S.J. Kim, S. Hu, C. Uher, T. Hogan, B. Huang, J.D. Corbett, M.G. Kanatzidis, J. Solid State Chem. 153, 321 (2000)

14. J.H. Kim, N.L. Okamoto, K. Kishida, K. Tanaka, H. Inui, Acta Mater. 54, 2057 (2006)

15. J.H. Kim, N.L. Okamoto, K. Kishida, K. Tanaka, H. Inui, J. Appl. Phys. 102, 094506 (2007)

16. W. Carrillo-Cabrera, H. Borrmann, S. Paschen, M. Baenitz, F. Steglich, Y. Grin, J. Solid State Chem. 178, 715 (2005)

17. F.M. Grosche, H.Q. Yuan, W. Carrillo-Cabrera, S. Paschen, C. Langhammer, F. Kromer, G. Sparn, M. Baenitz, Y. Grin, F. Steglich, Phys. Rev. Lett. 87, $247003(2001)$

18. B.H. Toby, J. Appl. Crystallogr. 34, 210 (2001)

19. A.C. Larson, R.B.V. Dreele, Los Alamos National Laboratory Report LAUR pp. 86-748 (1994)

20. U. Aydemir, C. Candolfi, H. Borrmann, M. Baitinger, A. Ormeci, W. Carrillo-Cabrera, C. Chubilleau, B. Lenoir, A. Dauscher, N. Oeschler, F. Steglich, Y. Grin, Dalton Trans. 39, 1078 (2010)

21. A. Grytsiv, N. Melnychenko-Koblyuk, N. Nasir, P. Rogl, A. Saccone, H. Schmid, Int. J. Mater. Res. 100, 189 (2009)

22. Y. Li, J. Chi, W. Gou, S. Khandekar, J.H. Ross, Jr., J. Phys.: Condens. Matter 15, 5535 (2003) 
23. X. Yan, E. Bauer, P. Rogl, S. Paschen, Phys. Rev. B 87, 115206 (2013)

24. U. Aydemir, C. Candolfi, A. Ormeci, M. Baitinger, N. Oeschler, F. Steglich, , Y. Grin, J. Phys.: Condens. Matter 26, 485801 (2014)

25. X. Shi, J. Yang, S. Bai, J. Yang, H. Wang, M. Chi, J.R. Salvador, W. Zhang, L. Chen, W. Wong-Ng, Adv. Funct. Mater. 20(5), 755 (2010)

26. S.Y. Rodriguez, L. Saribaev, J.H. Ross, Jr., Phys. Rev. B 82, $064111(2010)$

27. C. Candolfi, A. Ormeci, U. Aydemir, M. Baitinger, N. Oeschler, Y. Grin, F. Steglich, Phys. Rev. B 84 (2011)

28. I. Zerec, A. Yaresko, P. Thalmeier, Y. Grin, Phys. Rev. B 66, $045115(2002)$

29. D. Schopf, H. Euchner, H.R. Trebin, Phys. Rev. B 89, 214306 (2014)

30. N. Ashcroft, N. Mermin, Solid State Physics (Saunders College, Philadelphia, 1976)

31. I. Zerec, W. Carrillo-Cabrera, V. Voevodin, J. Sichelschmidt, F. Steglich, Y. Grin, A. Yaresko, S.I. Kimura, Phys. Rev. B 72, 045122 (2005)

32. T. Rachi, M. Kitajima, K. Kobayashi, F. Guo, T. Nakano, Y. Ikemoto, K. Kobayashi, K. Tanigaki, J. Chem. Phys. 123, 074503 (2005)

33. H.Q. Yuan, F.M. Grosche, W. Carrillo-Cabrera, V. Pacheco, G. Sparn, M. Baenitz, U. Schwarz, Y. Grin, F. Steglich, Phys. Rev. B 70, 174512 (2004) 